\title{
AN APPROACH TO THE POLYGONAL KNOT PROBLEM USING PROJECTIONS AND ISOTOPIES
}

\author{
BY \\ L. B. TREYBIG
}

\begin{abstract}
The author extends earlier work of Tait, Gauss, Nagy, and Penney in defining and developing properties of (1) the boundary collection of a knot function, and (2) simple sequences of knot functions or boundary collections. The main results are (1) if two knot functions have isomorphic boundary collections then the knots they determine are equivalent, and (2) if two knot functions determine equivalent knots, then the given functions (their boundary collections) are the ends of a simple sequence of knot functions (boundary collections). Matrices are also defined for knot functions.
\end{abstract}

1. Introduction. In [15] Tait considered the idea of associating with an oriented polygonal knot $M$ in regular position a "word" $W(M)$. For a trefoil knot whose projection double points are in order $a, b, c$ such a word could be $a b^{-1} c a^{-1} b c^{-1}$. Tait observed various properties of such words, and used them as an aid in developing quite an extensive set of knot tables. For example, he utilized the fact that between a letter and its inverse there must be an even number, perhaps zero, of other letters. He also recognized that the complementary domains of a knot projection can be colored in a "checker board" pattern (see [16]).

Earlier Gauss in [3] had studied similar finite sequences in connection with knot projections and had actually conjectured the even number property. In [8] Nagy proved Gauss' conjecture, and observed that a knot projection could be colored alternately with two colors $a, b$ such that as a point moved from one section through a crossing point into another section, the colors of the sections changed. Nagy also defined simple closed curves of the same color to be cycles.

In [10] Penney studied "words" for knots and defined certain "admissible" operations on words. He showed that if an admissible operation is performed on a word $W$ of a knot $K$ to form a new word $W^{\prime}$ then there is an equivalent knot $K^{\prime}$ whose word is $W^{\prime}$. Penney also proved an isomorphism theorem for a pair of knots, where one of them has a prime word, and the other's word can be changed by a finite sequence of admissible operations to an isomorphic word. A word $W$ is prime if $W$ cannot be written as $A B C$ where $B$ and $A \cup C$ are nonvoid, and where if $x^{e} \in B$ then $x^{-e} \in B$.

Received by the editors February 10, 1970.

AMS 1969 subject classifications. Primary 1188; Secondary 1184.

Key words and phrases. Knot function, words, boundary collection, $m$ by $n$ transformation, simple transformation, simple sequence, boundary collections, piecewise linear homeomorphism, simplicial isotopy. 
In [17] Treybig shows that if knots have isomorphic prime words then there is a certain unique correspondence between the complementary domains of their projections. In [16] Treybig characterizes those words in $2 n$ letters that are words for knot projections. Marx uses a condition of Mac Lane to give a simpler characterization in [5].

In the present paper the author combines the ideas of Penney's paper above and his own characterization paper, and associates with an oriented knot in regular position not only a word but an object called a "boundary collection". A notion of isomorphism and also "admissible" operations are defined for these. The first theorem says that if two knots $K, L$ have isomorphic boundary collections, then they are isomorphic in the sense that there is a piecewise linear homeomorphism of $E^{3}$ onto $E^{3}$ taking $K$ onto $L$. Theorem 8 yields that if two knots $K, L$ are isomorphic under an orientation-preserving homeomorphism of the space, then one may be obtained from the other by a certain finite sequence of "simple" moves and a corresponding sequence is obtained for the boundary collections. The importance of the boundary collection is that nearly all essential information about a given knot is "stored" in this object. Such collections can be given matrix representations, so it is evident that these would be highly useful in a study of knots by computer for example.

The main obstacle in the path of making this approach to knots complete is in not being able to show something like the following conjecture:

"If a piece of string in the shape of a simple closed curve can be moved in space so as to form at different times two knots in regular position whose projections have no more than $n$ crossing points, then the string can be moved from one configuration to the other in small steps so that no projection has more than $2 n$ crossing points."

In a later paper the author has some partial results on this problem, but no solution. The problem seems very difficult.

A student of the author, John Martin, has been able to show that if the above conjecture is true, then a modification of the ideas above yields a complete solution to various problems about invertible knots, amphicherial knots, etc. He also obtains certain results in connection with the word problem for Wirtinger presentations.

2. Definitions and notation. A polygonal knot (=knot) is a polyhedral simple closed curve in $E^{3}$. A knot $K$ is in regular position relative to a plane $W$ provided $K$ is a union of straight line intervals $A_{1} A_{2}, A_{2} A_{3}, \ldots, A_{n} A_{1}$, where the projection $\pi_{w}(K)$ into $W$ is at most two-to-one, and $x \in K$ and $\pi_{w}(x)=\pi_{w}\left(A_{p}\right)$ implies $x=A_{p}$. The points where the projection is two-to-one are crossing points or double points of the projection. $K$ in regular position means relative to the $x y$ plane. $K$ written as $A_{1} A_{2} \cdots A_{n}$ means each $A_{p} A_{p+1}$ is a straight line interval (if $p=n$, let $p+1 \equiv 1$ ), and card $\left(A_{p} A_{p+1} \cap A_{q} A_{q+1}\right)>1$ implies $p=q$. 
If $f:[0,1) \rightarrow K$ is continuous, one-to-one, and onto, with $K$ a knot, then $f$ is a knot function. Given $f:[0,1) \rightarrow K$ a knot function, $K$ in regular position and $0 \leqq a_{1}<a_{2}<\cdots<a_{2 n}<1$ the elements $x$ of $[0,1)$ so that $\pi f(x)$ is a double point of $\pi(K)$, then the $a_{i}$ are called double points of $f$, and if $\pi f\left(a_{i}\right)=\pi f\left(a_{j}\right)(i \neq j)$ let $a_{i}=m\left(a_{j}\right)$ (the mate). Under the above conditions the word of $f$ is

$$
W(f)=\left(\pi f\left(a_{1}\right)\right)^{e_{1}}\left(\pi f\left(a_{2}\right)\right)^{e_{2}} \cdots\left(\pi f\left(a_{2 n}\right)\right)^{e_{2 n}}
$$

where if $a_{j}=m\left(a_{i}\right)$ then $e_{i}=1$ (or is omitted) and $e_{j}=-1$ if $f\left(a_{i}\right)$ has a larger $z$ coordinate than $f\left(a_{j}\right)$. To define the word of the knot function $f:[0,1) \rightarrow K$ relative to plane $P$ and a given side $S$ of $P$ assume $K$ in regular position relative to $P$ and define in the obvious way making use of a transformation $T$ having the property that $T(P)$ is the $x y$ plane and the positive $z$ axis is a subset of $T(S)$, where $T$ is a rotation of $E^{3}$ followed by a translation.

Given $f:[0,1) \rightarrow K$ a knot function, with $K$ in regular position, the boundary collection $C(f)$ of $f$ is $\{D$ : there is a complementary domain $U$ of $\pi K$ so that $d \in D$ provided $d=\left(\pi f\left(a_{j}\right)\right)^{e_{i}}\left(\pi f\left(a_{i+1}\right)\right)^{e_{i+1}}$, where $\left.\pi f\left(\left[a_{i}, a_{i+1}\right]\right) \subset \operatorname{Bd}(U)\right\}$. Let $\left[a_{2 n}, a_{1}\right]$ denote $\left[a_{2 n}, 1\right) \cup\left[0, a_{1}\right]$. We extend the above definition to define boundary collection $C(f)$ of $f$ relative to plane $P$ and a given side $S$ of $P$.

If (1) $f:[0,1) \rightarrow K$ and $g:[0,1) \rightarrow L$ are knot functions with $K, L$ in regular position and (2) $m, n \in\{0,1,2,3, \ldots\}$ then $g$ has been obtained from $f$ by an $m$ by $n$ transformation $T$ (write $f \stackrel{T}{\rightarrow} g$ ) provided there exists $S \subset[0,1$ ) and a polyhedral disk $D$ with triangulation $T_{D}$ so that (0) $\pi$ is $1-1$ on $D$, (1) the simple closed curve Bd $(D)$ is the union of $\operatorname{arcs} A X B, A Y B$, (2) $f(S)=A X B$ and $g(S)=A Y B$, (3) $\pi A X B$ (alternately, $\pi A Y B$ ) contains exactly $m$ (alternately, $n$ ) double points of $\pi K$ (alternately, $\pi L$ ), (4) neither $A$ nor $B$ is a double point of $\pi K$ nor of $\pi L,(5) K \cap D=A X B$ and $L \cap D=A Y B$, (6) $f(x)=g(x)$ if $x \in[0,1)-S$, and (7) if the interval $G$ is a subset of $K-D$ and $V$ is a 1-simplex in $T$ then card $(\pi G \cap \pi V)$ is no more than 1 .

We define some special types of $m$ by $n$ transformations.

(a) Type 0. This is where (1) $m=n$, (2) if $C$ is a component of $\pi K \cap \operatorname{Int}(\pi(D))$, then $\bar{C} \cap(\pi \operatorname{Bd}(D))=\{\pi x, \pi y\}$ where $x \in \operatorname{seg} A X B$ and $y \in \operatorname{seg} A Y B$ or $\bar{C} \cap$ $(\pi \operatorname{Bd}(D))=\{\pi A, \pi B\}$, and (3) there are no double points of $\pi K$ in Int $\pi(D)$.

(b) Types I and $\mathrm{I}^{\prime}$. When (1) there is exactly one component $C$ of $\pi K \cap$ Int $\pi(D)$, where $C$ contains no double point of $\pi K$, and (2) $\bar{C} \cap(\pi \mathrm{Bd}(D))=\{\pi x, \pi y\}$ where $x=A$ or $B$ and $y \in \operatorname{seg} A Y B$, we say $g$ (alternately $f$ ) has been obtained from $f$ (alternately $g$ ) by a Type I (alternately Type $\left.I^{\prime}\right)$ transformation $(m=0, n=1)$.

(c) Types II and II'. When (1) there is exactly one component $C$ of $\pi K \cap \operatorname{Int} \pi(D)$, where $C$ contains no double points of $\pi K$, and (2) both points of $\bar{C}-C$ lie in $\pi$ seg $A Y B$, then we say $g$ (alternately, $f$ ) has been obtained from $f$ (alternately, $g$ ) by a Type II (II') transformation.

(d) Type III. When (1) there is exactly one component $C$ of $\pi K \cap \operatorname{Int} \pi(D)$ and there is exactly one crossing point of $\pi K$ in $C$, and (2) $\bar{C}-C$ has exactly two points 
in $\pi(\operatorname{seg} A X B)$ and exactly two in $\pi(\operatorname{seg} A Y B)$, then we say $g$ has been obtained from $f$ by a Type III transformation. Here $m=n=2$.

Now suppose $f:[0,1) \rightarrow K$ and $g:[0,1) \rightarrow L$ are knot functions with words

$$
W(f)=\left(\pi\left(f\left(a_{1}\right)\right)\right)^{e_{1}} \cdots\left(\pi f\left(a_{2 n}\right)\right)^{e_{2 n}}
$$

and

$$
W(g)=\left(\pi\left(g\left(b_{1}\right)\right)\right)^{d_{1}} \cdots\left(\pi g\left(b_{2 m}\right)\right)^{d_{2 m}}
$$

respectively. We say $W(f)$ is isomorphic to $W(g)(W(f) \cong W(g))$ if and only if (1) $m=n,(2)$ there is a cyclic permutation $h$ of $1, \ldots, 2 n$ so that $\pi f\left(a_{i}\right)=\pi f\left(a_{j}\right)$ if and only if $\pi g\left(b_{h(i)}\right)=\pi g\left(b_{h(j)}\right)$ and $d_{h(i)}=e_{i}$ and $d_{h(j)}=e_{j}$. We say $C(f)$ is isomorphic to $C(g)(C(f) \cong C(g))$ if and only if (1) $W(f) \cong W(g)$ (with $h$ as above) and (2) there is a 1-1 correspondence $K \rightarrow K^{\prime}$ between the elements of $C(f)$ and those of

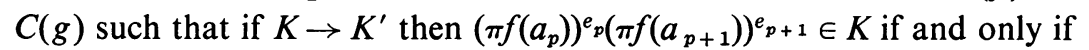

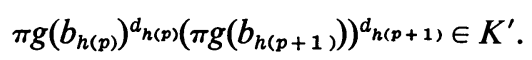

The $m$ by $n$ transformation of Types $0, I_{1}, I^{\prime}$, II, II', III are called simple transformations. Also if $f_{0}, f_{1}, \ldots, f_{n}$ is a sequence of knot functions and there exist simple transformations $T_{1}, \ldots, T_{n}$ so that $T_{p}: f_{p-1} \rightarrow f_{p}$ then we call

$$
f_{0} \stackrel{T_{1}}{\longrightarrow} f_{2} \stackrel{T_{2}}{\longrightarrow} \cdots \stackrel{T_{n}}{\longrightarrow} f_{n}
$$

a simple sequence of knot functions. Furthermore, if $f_{0}, f_{1}, \ldots, f_{n}$ is a sequence of knot functions, we shall say $C\left(f_{0}\right) \stackrel{s_{1}}{\rightarrow} C\left(f_{1}\right) \rightarrow \ldots \stackrel{s_{n}}{\rightarrow} C\left(f_{n}\right)$ is a simple sequence of boundary collections provided it is true that, if $0 \leqq p \leqq n-1$, then either (a) $C\left(f_{p}\right)$ $\cong C\left(f_{p+1}\right)$, or (b) there exists knot functions $g, h$ so that $C(g) \cong C\left(f_{p}\right), C(h) \cong C\left(f_{p+1}\right)$, and $h$ is obtained from $g$ by a simple transformation $T$. In case (b) we also say that $S_{p}$ is of the same type as $T$.

3. A basic theorem. In this section, we give a proof of the theorem which shows that the boundary collection of a knot function retains a great deal of information about the function.

THEOREM 1. Suppose that each of $f:[0,1) \rightarrow K$ and $g:[0,1) \rightarrow L$ is a knot function. Then, if $C(f) \cong C(g)$ there is a piecewise linear (p.l.) homeomorphism $h$ from $E^{3}$ onto $E^{3}$ such that $(1) h(K)=L,(2)$ if $x$ and $y$ are distinct elements of $K$ so that $\pi(x)=\pi(y)$, then interval $x y$ has the property that $h(x y)$ is an interval from $h(x)$ to $h(y)$, and (3) $h(f)$ induces the same orientation on $L$ as $g$. Furthermore, if the element of $C(f)$ corresponding to the unbounded complementary domain of $\pi K$ also corresponds to the analogous element of $C(g)$ under the isomorphism $C(f) \cong C(g)$, then $h$ may be chosen so that $h(\pi x)=\pi h(x)$.

Proof. Following Penney in Lemma 4 of [10], let the double points of $f$ be $a_{1}, \ldots, a_{2 n}$ and those of $g$ be $b_{1}, \ldots, b_{2 n}$. Since $C(f) \cong C(g)$ implies that $W(f)$ $\cong W(g)$, let us suppose that $a_{1} \sim b_{1}$ under this correspondence. 
Let $U$ denote the complementary domain of $\pi K$ which corresponds to the unbounded complementary domain of $\pi L$ under the correspondence $C(f) \cong C(g)$; and suppose the worst case, that $U$ is bounded. There is a polygonal arc $p q$ with $p$ in the unbounded complementary domain of $\pi K$ and $q$ in $U$ such that (1) $p q$ contains no double points of $\pi K$, (2) if $S=\left[a_{i}, a_{i+1}\right]$ then $\pi f(S)$ contains at most one point of $p q$ and (3) if $V$ is a complementary domain of $\pi K$, then $V \cap p q$ is connected. Let $r_{0}, r_{1}, \ldots, r_{m-1}$ denote the points of $\pi K \cap p q$ in their natural order from $p$ to $q$, and let $\left(c_{0}, d_{0}\right), \ldots,\left(c_{m-1}, d_{m-1}\right)$ denote open subintervals of $[0,1)$ so that $r_{i} \in \pi f\left(\left(c_{i}, d_{i}\right)\right)$ and

$$
\mathrm{Cl}\left(\bigcup_{i=0}^{m-1}\left(c_{i}, d_{i}\right)\right) \subset[0,1)-\left\{a_{1}, \ldots, a_{2 n}\right\} .
$$

Using Corollary 1 of Sanderson [12], we may construct knot functions $k_{0}=f, k_{1}$, $\ldots, k_{m}$ and homeomorphisms $h_{1}, \ldots, h_{m}$ such that for each integer $p, 0 \leqq p<m-1$,

(1) $k_{p+1}(x)=k_{p}(x)$ if $x \in[0,1)-\left(c_{p}, d_{p}\right)$,

(2) $\pi\left(k_{p+1}\left(\left[c_{p}, d_{p}\right]\right) \cup k_{p}\left(\left[c_{p}, d_{p}\right]\right)\right)$ is a polygonal simple closed curve containing $\pi k_{p}\left([0,1)-\left(c_{p}, d_{p}\right)\right)$ in its interior, and

(3) $h_{p}$ is a p.l. homeomorphism from $E^{3}$ onto $E^{3}$ that takes $k_{p}[0,1)$ onto $k_{p+1}[0,1)$ in such a way that it is the identity on any straight line interval joining points of the form $k_{p}\left(a_{i}\right)$ and $k_{p}\left(a_{j}\right)$, where $m\left(a_{i}\right)=a_{j}(i \neq j)$, and

(4) $W\left(k_{0}\right)=W\left(k_{1}\right)=\cdots=W\left(k_{m}\right)$ and $C\left(k_{0}\right)=C\left(k_{1}\right)=\cdots=C\left(k_{m}\right)$.

We now define a p.l. homeomorphism $w$ of the $x y$ plane onto itself. First consider triangulations $R_{1}$ and $R_{2}$ of $\pi k_{m}([0,1))$ and $\pi L$, respectively, such that, if $1 \leqq i \leqq 2 n$, then $\pi k_{m}\left(a_{i}\right)$ is a 0 -simplex of $R_{1}$ and $\pi g\left(a_{i}\right)$ is a 0 -simplex of $R_{2}$. Let $t$ denote a p.l. homeomorphism from $\pi k_{m}([0,1))$ onto $\pi L$ so that, for each $i=1, \ldots, 2 n$, $t \pi k_{m}\left(a_{i}\right)=\pi g\left(a_{i}\right)$ and $t \pi k_{m}\left(\left[a_{i}, a_{i+1}\right]\right)=\pi g\left(\left[a_{i}, a_{i+1}\right]\right)$.

Now suppose $V$ is a complementary domain of $\pi k_{m}([0,1))$ and $V^{\prime}$ is its corresponding domain under the correspondence $C\left(k_{m}\right) \cong C(g)$. We give the argument here in the case $V$ is bounded, but the unbounded case is also easily handled. Let $d_{1}, \ldots, d_{j}$ denote the double points of $\pi k_{m}([0,1))$ on $\mathrm{Bd} V$, and let $d_{1}^{\prime}, \ldots, d_{j}^{\prime}$ denote those of $\pi L$ on $\mathrm{Bd} V^{\prime}$, where $\pi k_{m}\left(a_{t}\right)=d_{s}$ implies that $\pi g\left(a_{t}\right)=d_{s}^{\prime}$. Now suppose $P \in V$ and consider polygonal arcs $P D_{1}, P D_{2}, \ldots, P D_{N}$ such that (a) $\left\{D_{1}, \ldots, D_{N}\right\}=\left\{d_{1}, \ldots, d_{j}\right\}$, (b) $P D_{p}-D_{p} \subset V, p=1, \ldots, N$, (c) if $\pi k_{m}\left(\left[a_{i}, a_{i+1}\right]\right)$ $\subset \mathrm{Bd} V$ and $m\left(a_{i}\right)=a_{i+1}$, then there exist integers $p, p+1$ so that $\pi k_{m}\left(\left(a_{i}, a_{i+1}\right)\right)$ $\subset$ Int $\left(P D_{p} \cup P D_{p+1}\right)$, (d) if $\pi k_{m}\left(\left[a_{i}, a_{i+1}\right]\right) \subset \mathrm{Bd} V$ and $m\left(a_{i}\right) \neq a_{i+1}$, then there exist integers $p, p+1$ so that $\left\{D_{p}, D_{p+1}\right\}=\left\{\pi k_{m}\left(a_{i}\right), \pi k_{m}\left(a_{i+1}\right)\right\}$, (e) the arcs $P D_{p}$ and $P D_{q}(p \neq q)$ intersect in no points besides endpoints, and (f) no $d_{i}=D_{p}$ for more that two indices $p$, and if $d_{i}$ does not separate $\mathrm{Bd} V$ then $d_{i}=D_{p}$ for a unique $p$. We also pick $P^{\prime}$ and $V^{\prime}$ and a corresponding set of polygonal $\operatorname{arcs} P^{\prime} D_{1}^{\prime}, \ldots, P^{\prime} D_{n}^{\prime}$ such that

(1) (a)-(f) hold for the primed quantities, and

(2) $D_{p}=d_{i}$ implies $D_{p}^{\prime}=d_{i}^{\prime}$. 
Now let $t_{V}:\left(\mathrm{Bd} V \cup \sum P D_{p}\right) \rightarrow\left(\mathrm{Bd} V^{\prime} \cup \sum P^{\prime} D_{p}^{\prime}\right)$ be an onto p.l. homeomorphism such that (a) $t_{V}(P)=P^{\prime}, t_{V}\left(D_{p}\right)=D_{p}^{\prime}(p=1, \ldots, N)$, and $t_{V}\left(P D_{p}\right)=P^{\prime} D_{p}^{\prime}(p=1$, $\ldots, N)$, and (b) $t_{V}$ agrees with $t$ on $\mathrm{Bd} V$.

For each component $C$ of $V-\sum P D_{p}$ bounded by two consecutive arcs $P D_{p}$, $P D_{p+1}$ and an arc (or simple closed curve) $D_{p} D_{p+1}$, we extend our transformation $t_{V}$ to a p.l. homeomorphism $t_{C}$ carrying $\bar{C}$ onto the corresponding set in $\bar{V}^{\prime}$. Each set $\bar{C}$ is either a polyhedral 2-cell or one with two boundary points identified, so the mapping is not hard to extend. The mappings $t_{C}$ combine to define a p.l. homeomorphism $l_{V}: \bar{V} \rightarrow \bar{V}^{\prime}$ so that $l_{v}(x)=t(x)$ if $x \in \mathrm{Bd} V$.

We now define $w$ from the $x y$ plane onto the $x y$ plane so that if $x$ is an element of $\bar{V}$, where $V$ is a complementary domain of $\pi k_{m}([0,1))$, then $w(x)=l_{V}(x)$. Let $T_{1}$ denote a triangulation of the $x y$ plane so that (1) $w$ is linear on each simplex $s$ of $T_{1}$, (2) each $\pi k_{m}\left(a_{i}\right)$ is a 0 -simplex of $T_{1},(3)$ the 1 -skeleton of $T_{1}$ contains $\pi k_{m}([0,1))$, and (4) if $x \in k_{m}([0,1))$ and is not a subset of an open interval lying in that set, then $\pi x$ is a 0 -simplex of $T_{1}$; and if $y \in g([0,1))$ and is not a subset of an open interval lying in that set, then $w^{-1}(\pi(y))$ is a 0 -simplex of $T_{1}$.

We now define a homeomorphism $k$ from $k_{m}([0,1))$ onto $g([0,1))$. For each point $k_{m}\left(a_{i}\right)$ let $k$ of $\left(k_{m}\left(a_{i}\right)\right)=g_{m}\left(a_{i}\right)$. Thus on each line parallel to the $z$ axis which contains two points of $k_{m}[0,1)$, we have defined $k$ for those two points, so we extend linearly. For every other such line which passes through a 0 -simplex of $T_{1}$ and a point of $k_{m}[0,1)$, say $k_{m}(x)$, let $k\left(k_{m}(x)\right)=\pi^{-1}(w(x)) \cap L$ and let $k$ on that line simply denote a translation. For every other such line through a zero simplex $s$ of $T_{1}$ let $T_{1}(l)$ be a translation of $l$ so that it contains $w(s) . k$ is now defined on each line parallel to the $z$ axis containing a 0 -simplex of $T_{1}$. We extend the function now on each triangular based cylinder to find $k$. $h$ is defined to be $k h_{m} h_{m-1} \cdots h_{1}$.

4. Approximations. In this section, we prove a theorem on approximations which is needed in the later sections.

Following Sanderson [12] we assume that all simplexes are closed and that if $V_{0}, \ldots, V_{n}$ is a set of points, then $\left\langle V_{0}, \ldots, V_{n}\right\rangle$ will denote the closed convex hull of $\left\{V_{0}, \ldots, V_{n}\right\}$ (this is the $n$ simplex whose vertices are $V_{0}, \ldots, V_{n}$ provided $V_{0}, \ldots, V_{n}$ is an affinely independent set).

TheOREM 2. Suppose $T$ is a finite m-complex in Euclidean $n$-space $E^{n}$. Then, there is a positive number $\varepsilon$ such that if $V_{0}, V_{1}, \ldots$ denote the 0 -simplexes of $T$ and $V_{0}^{\prime}, V_{1}^{\prime}, \ldots$ denote $\varepsilon$ approximations of these, respectively, then the collection $T^{\prime}$ to which $t$ belongs if and only if there is a simplex $\sigma=\left\langle V_{n_{i}}, \ldots, V_{n_{j}}\right\rangle$ of $T$ such that $t=$ $\left\langle V_{n_{i}}^{\prime}, \ldots, V_{n_{i}}^{\prime}\right\rangle\left(\right.$ denote $t$ by $\sigma^{\prime}$ ) is also a finite m-complex such that (1) if $\sigma$ is a $k$ complex of $T$ then $\sigma^{\prime}$ is a $k$-complex of $T^{\prime}$, (2) if $\sigma_{1}, \sigma_{2} \in T$ and do not intersect, then $\sigma_{1}^{\prime}$ and $\sigma_{2}^{\prime}$ do not intersect, and (3) if $\sigma_{1}, \sigma_{2}, \sigma \in T$ and $\sigma_{1} \cap \sigma_{2}=\sigma$ then $\sigma_{1}^{\prime} \cap \sigma_{2}^{\prime}=\sigma^{\prime}$.

Proof. The remainder of the theorem is established easily from part (2). Let $\varepsilon>0$ be less than half the distance between any two disjoint simplexes of $T$. 
Suppose that $\sigma_{1}=\left\langle V_{0}, \ldots, V_{i}\right\rangle$ and $\sigma_{2}=\left\langle W_{0}, \ldots, W_{j}\right\rangle$ are disjoint simplexes of $T$, but that $P \in \sigma_{1}^{\prime} \cap \sigma_{2}^{\prime}$. Then $P=\sum_{0}^{i} a_{p} V_{p}^{\prime}=\sum_{0}^{j} b_{p} W_{p}^{\prime}$, where $\sum_{0}^{i} a_{p}=1, a_{p} \geqq 0$; $\sum_{0}^{j} b_{p}=1, b_{p} \geqq 0$. Thus, we have

$$
\begin{aligned}
2 \varepsilon & <\left\|\sum_{0}^{i} a_{p} V_{p}-\sum_{0}^{j} b_{p} W_{p}\right\|=\left\|\sum_{0}^{i} a_{p} V_{p}-\sum_{0}^{i} a_{p} V_{p}^{\prime}+\sum_{0}^{j} b_{p} W_{p}^{\prime}-\sum_{0}^{j} b_{p} W_{p}\right\| \\
& \leqq \sum_{0}^{i} a_{p}\left\|V_{p}-V_{p}^{\prime}\right\|+\sum_{0}^{j} b_{p}\left\|W_{p}^{\prime}-W_{p}\right\| \leqq 2 \varepsilon,
\end{aligned}
$$

which yields a contradiction.

5. $m$ by $n$ transformations. In this section we prove several theorems about $m$ by $n$ transformations, the first of which says that an $m \times n$ transformation can be considered as a sequence of small steps, with a bound on crossing.

THEOREM 3. Suppose $f:[0,1) \rightarrow K$ and $g:[0,1) \rightarrow L$ are knot functions such that $g$ has been obtained from $f$ by an $m$ by $n$ transformation $T$ and that $D$ is a polyhedral disk, $S \subset[0,1)$, arc $A X B=f(S)$, arc $A Y B=g(S)$, and $\mathrm{Bd}(D)=A X B Y A$, where $D$, $S, A X B$ and $A Y B$ are as in the definition of $m$ by $n$ transformation. Then, there is $a$ sequence $g_{0}, g_{1}, \ldots, g_{q}$ of knot functions such that (1) $g_{0}=f, g_{q}=g,(2) g_{p}(x)=f(x)$ for $x \in[0,1)-S$ and $g_{p}(S) \subset D$ for $p=0,1, \ldots, q$, (3) $g_{p}$ is obtained from $g_{p-1}$ by a simple transformation for $p=1, \ldots, q$, (4) if $D_{p}$ is the subdisk of $D$ bounded by $g_{0}(S) \cup g_{p}(S)$ for $p=1, \ldots, q$, then $D_{p} \subset D_{p+1}$ for $p=1, \ldots, q-1$, and (5) if $c\left(g_{p}\right)$ denotes the number of crossing points of $\pi g_{p}([0,1))$ and $c$ denotes the number of crossing points of $\pi L$ in Int $(\pi D)$, then $c\left(g_{p}\right) \leqq 2 c+\sup \{c(f), c(g)\}$ for $p=0,1, \ldots, q$.

Proof. We proceed by induction on the number $c$. Let $T_{D}$ denote a triangulation of $D$ as guaranteed in the definition of $m$ by $n$ transformation.

Case 1. $c=0$. We note that (1) if there are no components of $\pi(L) \cap \pi(\operatorname{Int}(D))$, or (2) if every component of $\pi(L) \cap \pi(\operatorname{Int}(D))$ has exactly one endpoint on $\pi \operatorname{seg} A X B$ and exactly one endpoint on $\pi \operatorname{seg} A Y B$, or (3) there is exactly one component of $\pi(L) \cap \pi(\operatorname{Int}(D))$ whose endpoints are $\pi A$ and $\pi \dot{B}$, respectively, then $T$ is a transformation of Type 0 and the theorem is trivially true. We therefore proceed in this case by induction on the number of components of $\pi(L) \cap \pi(\operatorname{Int}(D))$, assuming there is at least one such component which does not satisfy the above.

We first consider a slight adjustment of $\operatorname{seg} A X B$ into Int $(D)$ so as to form a new arc $A X_{1} B$, where (1) there is a knot function $f_{1}:[0,1) \rightarrow(K-A X B) \cup A X_{1} B$ so that $f(x)=f_{1}(x)$ for $x \in[0,1)-S$, and (2) if $D_{1}$ denotes the subdisk of $D$ bounded by $A Y B \cup A X_{1} B$ and $C$ is a component of $\pi \operatorname{Int}\left(D_{1}\right) \cap \pi f_{1}([0,1))$, then $C$ has one endpoint on $\pi \operatorname{seg} A X_{1} B$ and one endpoint on $\pi \operatorname{seg} A X B$. Thus, there is a Type 0 transformation $T_{1}: f \rightarrow f_{1}$, and $f_{1}, g$, and $\mathrm{Cl}\left(D-D_{1}\right)$ satisfy the same induction hypothesis.

Suppose some component $C$ of $\pi L \cap \pi \operatorname{Int}\left(D_{1}\right)$ has the property that the endpoints $P, Q$ of $C$ lie in $\pi A X B$ (the case for $A Y B$ is handled analogously). It follows that there is such a component $C^{\prime}=\operatorname{seg} P^{\prime} Q^{\prime}$ whose closure separates no other such component from $\pi \operatorname{seg} A Y B$ in $\pi D_{1}$. Let $T U$ denote a polygonal arc in 
$D_{1}$ such that (1) $T, U \in A X_{1} B$ and seg $T U \subset$ Int $D_{1}$, (2) $\pi T U$ separates $C^{\prime}$ from all other components of $\pi L \cap \pi \operatorname{Int}\left(D_{1}\right)$ in $\pi D_{1}$, and (3) there is a knot function $f_{2}:[0,1) \rightarrow\left(f_{1}([0,1))-\left(\right.\right.$ subarc $T U$ of $\left.\left.A X_{1} B\right)\right) \cup T U$ so that $f_{2}(x)=f_{1}(x)$ for $x \in\left[f_{1}^{-1}\left(f_{1}([0,1)) \cap f_{2}([0,1))\right)\right]$. We see that $f_{2}$ has been obtained from $f_{1}$ by transformation $T_{2}$ of Type II' (respectively, Type $\mathrm{I}$, if one of $P^{\prime}$ or $Q^{\prime}$ is $\pi A$ or $\pi B$ ).

We now apply our induction hypotheses to $f_{2}, g$ and the subdisk of $D$ bounded by $f_{2}(S) \cup g(S)$ to obtain a sequence $h_{0}=f_{2}, h_{1}, \ldots, h_{t}=g$ satisfying the conclusions of the theorem. Our desired sequence is then $f, f_{1}, h_{0}, h_{1}, \ldots, h_{t}$. Also note that $c\left(f_{1}\right)=c\left(f_{0}\right), c\left(h_{0}\right)=c(f)-i$ where $i=1$ or 2 . Then $c\left(h_{p}\right) \leqq 2 c+\sup \left\{c\left(h_{0}\right), c\left(h_{t}\right)\right\}$ $\leqq 2 c+\sup \{c(f), c(g)\}, p=0, \ldots, t$, and so if our sequence is then labeled $g_{0}, \ldots$, $g_{t+2}$, we have $c\left(g_{p}\right) \leqq 2 c+\sup \{c(f), c(g)\}, p=0, \ldots, t+2$.

Case 2. $c>0$. First we construct $f_{1}$ and $D_{1}$ as in the previous case. There is a component $C$ of $\pi\left(\operatorname{Int}\left(D_{1}\right)\right) \cap \pi(L)$ such that $\bar{C}$ contains two arcs $E F G$ and $H F J$ such that (1) $E F G$ crosses $H F J$ at $F$, (2) seg $E F G \cup H F J$ is open relative to $C$ and (3) $(E F G \cup H F J) \cap \mathrm{Bd}\left(\pi D_{1}\right)=E$ belongs to $\operatorname{seg} \pi A X_{1} B$ or to $\operatorname{seg} \pi A Y B$. We handle only the case $E \in \operatorname{seg} \pi A X_{1} B$, since the other case is handled analogously.

There exist points $P, Q, H_{1}, G_{1}, J_{1}$ of $D$ such that (1) $P$ and $Q$ occur in the order $A P Q B$ on arc $A X_{1} B,(2) \pi H_{1} \in \operatorname{seg} H F$ of arc $H F J, \pi G_{1} \in \operatorname{seg} F G$ of arc $E F G$, and $\pi J_{1} \in \operatorname{seg} F J$ of arc $H F J$, (3) there is a polygonal arc $P G_{1} Q$ containing $H_{1}, G_{1}$, and $J_{1}$ and which is a subset of $D_{1}$ such that (a) $\operatorname{seg} P G_{1} Q \subset \operatorname{Int}\left(D_{1}\right)$, (b) $\pi\left(P G_{1} Q\right)$ $\cap\left(\pi L \cap \pi \operatorname{Int}\left(D_{1}\right)\right)=\pi\left(H_{1} \cup G_{1} \cup J_{1}\right)$, and (c) if $P Q$ denotes the subarc of $A X_{1} B$ with endpoints $P, Q$, then there is a knot function $f_{2}:[0,1) \rightarrow\left(f_{1}([0,1))-P Q\right)$ $\cup P G_{1} Q$ so that $f_{2}(x)=f_{1}(x)$ for $x \in f_{1}^{-1}\left[f_{1}([0,1)) \cap f_{2}([0,1))\right]$. We then consider a new knot function $f^{\prime}$ and a sequence satisfying the conditions of the theorem for $f, f_{2}$, the sequence being $f, f_{1}, f^{\prime}, f_{2}$ where the construction of $f^{\prime}$ may be determined from Figure 1. The dotted interval denotes the change from $f_{1}$ to $f^{\prime}$. Here there exist transformations $T^{\prime}: f_{1} \rightarrow f^{\prime}$ and $T_{2}: f^{\prime} \rightarrow f_{2}$, where $T^{\prime}$ is Type II and $T_{2}$ is of Type III.

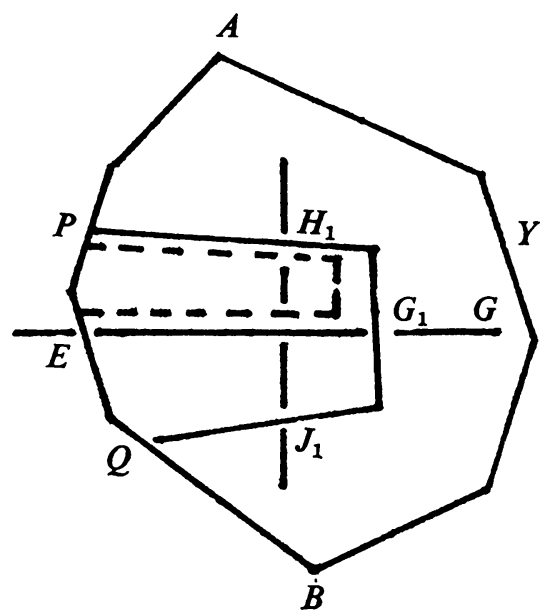

Figure 1 
Now, using the induction hypothesis on $f_{2}, g$ and the subdisk of $D$ bounded by $f_{2}(S) \cup g(S)$, we find a sequence $h_{0}=f_{2}, h_{1}, \ldots, h_{t}=g$ satisfying the conclusion of the theorem, where $c\left(h_{p}\right) \leqq 2(c-1)+\sup \{c(f), c(g)\} \leqq 2 c+\sup \{c(f), c(g)\}$. Also $c\left(f_{2}\right)=2+c(f) \leqq 2 c+\sup \{c(f), c(g)\}$, since $c \geqq 1$. Therefore, the desired sequence is $f, f_{1}, f^{\prime}, f_{2}, h_{1}, \ldots, h_{t}$.

THEOREM 4. If $f:[0,1) \rightarrow K$ and $g:[0,1) \rightarrow L$ are knot functions and $T: f \rightarrow g$ is an $m \times n$ transformation, then there is an orientation-preserving homeomorphism $h$ from $E^{3}$ onto $E^{3}$ taking $K$ onto $L$ and which is the identity on $K \cap L$.

Proof. Let arcs $A X B, A Y B$ and disk $D$ be in the definition of $m$ by $n$ transformation. Let $D^{\prime}$ denote a polyhedral disk so that $D-(A \cup B) \subset \operatorname{Int} D^{\prime}, D^{\prime} \cap K$ $=A X B$ and $D^{\prime} \cap L=A Y B$. Let $U$ denote a polyhedral open set containing $D^{\prime}-(A \cup B)$ so that $K-D^{\prime}=L-D^{\prime} \subset E^{3}-U$. By Corollary 1 of [12] there is a simplicial isotopy $h_{t}$ of $E^{3}$ onto $E^{3}$ which is (1) fixed outside $U$, (2) is invariant on $D^{\prime},(3)$ is the identity for $t=0$, and (4) takes $A X B$ onto $A Y B$ for $t=1$. The desired mapping is $h_{1}(x)$.

THeOREM 5. If $f:[0,1) \rightarrow K$ and $g:[0,1) \rightarrow L$ are knot functions and $T: f \rightarrow g$ is an $m$ by $n$ transformation of Type 0 , then $C(f)=C(g)$.

Proof. The proof is omitted.

6. Simplicial isotopies. We need to make several new definitions at this stage which are mainly restrictions of the definitions in [12], [13] to $E^{3}$.

$H\left(E^{3}\right)$ will denote the space of homeomorphisms of $E^{3}$ onto $E^{3}$ together with the compact-open topology. An element $h$ of $H\left(E^{3}\right)$ will be said to be piecewise linear if there is a rectilinear triangulation $T$ of $E^{3}$ such that $h$ is linear on each simplex of $T$. A rectilinear triangulation is one whose simplexes are points, straight line intervals, triangular disks or solid tedrahedra. Furthermore, if $\boldsymbol{P}$ is a polyhedron in such a triangulation $T$ and $f:|P| \rightarrow E^{3}$ then $f$ will be said to be piecewise linear if $f$ is linear on each simplex of some subdivision of $T$. If $G:|P| \times I \rightarrow E^{3}$ then $G$ will be called a piecewise linear isotopy if $G$ is continuous and each $G_{t}$ is a piecewise linear homeomorphism of $|P|$ into $E^{3}$. In addition, in the above situation $G$ will be called a simplicial isotopy if $G$ is a piecewise linear isotopy such that $G_{t}$ is linear on each simplex of some fixed subdivision of $P$ for all $t$ in $[0,1]$. Also if $G:|P| \times I \rightarrow E^{3}$ is a piecewise linear isotopy and $\varepsilon>0, G$ is called an $\varepsilon$-isotopy if the image of $p \times[0,1]$ has diameter less than $\varepsilon$ for each $p \in|P|$.

We now state a useful theorem due to Moise [6]. The advantage of this theorem is that it allows us to concentrate on the piecewise linear homeomorphisms.

THEOREM M. If some homeomorphism $\phi$ of 3-space $E^{3}$ upon itself preserves the orientation and transforms a given simple closed polygon $J$ into a given simple closed polygon $J^{\prime}$, then there is a piecewise linear homeomorphism with these properties. 
THEOREM 6. Suppose that each of $K$ and $L$ is a polygonal knot in regular position in $E^{3}$ and that $f$ is an orientation-preserving piecewise linear homeomorphism from $E^{3}$ onto $E^{3}$ such that $f(K)=L$. Then, there is a simplicial isotopy $\phi: K \times[0,1] \rightarrow E^{3}$ such that $\phi(x, 0)=x$ and $\phi(x, 1)=f(x)$ for $x \in K$.

Proof. This theorem has been proved by Graeub [6]. It may also be shown using the work of Sanderson in [12], [13], [14].

THEOREM 7. Suppose $K, L, f$ and $\phi$ are as in Theorem 6 and that $S$ is a geometric 2-sphere so that $\phi(K \times[0,1]) \subset$ Int $S$. Then, (1) if $R$ is a countable subset of $[0,1]$ and $U$ is an open subset of $S$, there is a plane $P$ tangent to $S$ at some point $A$ of $U$ such that if $t \in R$ then $\phi_{t}(K)$ is in regular position relative to $P$, and (2) there is a fixed integer $N$ such that if $Q$ is a plane tangent to $S$ and $\phi_{t}(K)$ is in regular position relative to $Q$, then the projection $\pi_{Q}\left(\phi_{t}(K)\right)$ has no more than $N$ crossings.

Proof. (1) For each $\phi_{t}(K)$ there is a closed nowhere dense subset $C_{t}$ of $S$ such that $\phi_{t}(K)$ is in regular position relative to the tangent plane $P$ of $S$ if and only if $P$ is tangent to $S$ at a point of $S-C_{t}$. Therefore, since $R$ is countable and $S$ is a Baire space, there is a point $A$ of $U$ such that if $P$ is a plane tangent to $S$ at $A$ and $t \in R$, then $\phi_{t}(K)$ is in regular position relative to $P$.

(2) Since $\phi$ is a simplicial isotopy there is a triangulation $T$ of $K$ with exactly $n$ 1 -simplexes such that if $t \in[0,1]$ and $s$ is a 1-simplex in $T$ then $\phi_{t}(s)$ is a 1-simplex in $\phi_{t}(K)$. If $s$ and $s^{\prime}$ are two 1-simplexes in $T$ then $\pi_{Q}\left(\phi_{t}(s)\right)$ and $\pi_{Q}\left(\phi_{t}\left(s^{\prime}\right)\right)$ can cross each other no more than once, so $\pi_{Q}\left(\phi_{t}(K)\right)$ has no more than $N=n^{2}$ crossing points.

7. Simple sequences. We now combine the results of some of the previous sections to prove the following:

THEOREM 8. Suppose that each of $g:[0,1) \rightarrow K$ and $h:[0,1) \rightarrow L$ is a knot function, and that $f$ is an orientation-preserving homeomorphism from $E^{3}$ onto $E^{3}$ such that $f(K)=L$, and $f(g)$ and $h$ induce the same orientation on $L$. Then, there is a simple sequence of knot functions

$$
g=g_{0} \stackrel{S_{1}}{\longrightarrow} g_{1} \stackrel{S_{2}}{\longrightarrow} \ldots \stackrel{S_{n}}{\longrightarrow} g_{n}=f(g),
$$

and thus a simple sequence of boundary collections

$$
C(g)=C\left(g_{0}\right) \stackrel{S_{1}}{\longrightarrow} C\left(g_{1}\right) \stackrel{S_{2}}{\longrightarrow} \ldots \stackrel{S_{n}}{\longrightarrow} C\left(g_{n}\right)=C(h) .
$$

Proof. We apply Theorem 6 to obtain a simplicial isotopy $\phi: K \times[0,1] \rightarrow E^{3}$ such that $\phi(x, 0)=x$ and $\phi(x, 1)=f(x)$. Let $T$ denote a triangulation of $K$ so that if $S \in T$ and $t \in[0,1]$ then $\phi_{t}$ is affine on $S$.

In order to simplify the statements involved in our proof we suppose without loss of generality that $\phi(K \times[0,1]) \subset$ Int $S$, where $S$ is a geometric 2-sphere tangent to the $x y$ plane at $(0,0,0)$ and where if $(x, y, z) \in \operatorname{Int}(S)$ then $z>0$. There is an open subset $U$ of $S$ containing $(0,0,0)$ so that if $A \in U$ and $P$ is a plane tangent to 
$S$ at $A$, then $K$ and $L$ are in regular position relative to $P$. Let $R$ denote the rationals in $[0,1]$.

By Theorem 2 and the compactness of $[0,1]$, there is an $\varepsilon>0$ so that if $t \in[0,1]$ and $T_{t}=\{\phi(s, t): s \in T\}$ then the conclusions of Theorem 2 follow for $2 \varepsilon$ approximations of the 0 -simplexes of $T_{t}$. Let $0<d<1$ be such that if $t, t^{\prime} \in[0,1]$ and $\left|t-t^{\prime}\right|<d$ and $S$ is a 0 -simplex of $T$, then $\left\|\phi_{t}(S)-\phi_{t^{\prime}}(S)\right\|<\varepsilon$.

We apply Theorem 7 to $g, h, \phi, R, S, U$ to find $A^{\prime} \in U$ so that (1) $E^{3}$ may be rotated slightly about the center of $S$ so as to move $A^{\prime}$ to $(0,0,0)$ and so that no 0 -simplex of $T_{i}(i=0,1)$ is moved as much as $\varepsilon / 2$, and (2) if $P$ denotes the plane tangent to $S$ at $A^{\prime}$ and $t \in R$, then $\phi_{t}(K)$ is in regular position relative to $P$. At this stage we assume for the moment that the point $A^{\prime}$ involved is $(0,0,0)$ itself.

Let $0=a_{0}<a_{1}<\cdots<a_{k}=1$ be a partition of $[0,1]$ so that (1) each $a_{p} \in R$ and (2) if $0 \leqq p<k$, then $\left|a_{p}-a_{p+1}\right|<d$. Let $V_{0}, \ldots, V_{q}$ denote the 0 -simplexes of $T$ in some cyclic order, and for each $p \in\{0, \ldots, k\}$ let $A_{0 p}, \ldots, A_{q p}$ denote $\varepsilon / 2$ approximations of $\phi\left(V_{0}, a_{p}\right), \ldots, \phi\left(V_{q}, a_{p}\right)$, respectively, such that if $J_{p}$ is the union of intervals $A_{0 p} A_{1 p}, A_{1 p} A_{2 p}, \ldots, A_{q p} A_{0 p}$ then (0) $A_{i p}=\phi\left(V_{i}, a_{p}\right)$ for $p=0, k$, (1) each $J_{p}$ is in regular position, and (2) if $0 \leqq p<k$ then $J_{p}$ and $J_{p+1}$ do not intersect and no point of $\left\{A_{0 p}, \ldots, A_{q p}, A_{0, p+1} \cdots A_{q, p+1}\right\}$ projects onto a double point of

$$
\pi\left(J_{p} \cup J_{p+1}\right) \text {. }
$$

Now suppose $0 \leqq p<k$ and let $A_{i}=A_{i p}, B_{i}=A_{i, p+1}$ for $i=1, \ldots, q$. Letting $J_{A}=J_{p}$ and $J_{B}=J_{p+1}$, the natural p.l. homeomorphisms $h_{A}: \phi\left(K, a_{p}\right) \rightarrow J_{A}, h_{B}$ : $\phi\left(K, a_{p+1}\right) \rightarrow J_{B}$ induce knot functions $g_{A}=h_{A} \phi_{a_{p}} g$ and $g_{B}=h_{B} \phi_{a_{p+1}} g$.

We now consider a knot function $g^{\prime}:[0,1) \rightarrow\left(J_{A}-A_{1} A_{2}\right) \cup A_{1} B_{1} \cup A_{2} B_{1}$ such that $g^{\prime}(x)=g_{A}(x)$ for $x \in[0,1)-g_{A}^{-1}\left(A_{1} A_{2}\right)$. We now find that the hypothesis of Theorem 3 holds for $g_{A}, g^{\prime}$ and the disk $A_{1} A_{2} B_{1}$, and obtain a sequence of functions $g_{A}=h_{0}, h_{1}, \ldots, h_{m}=g^{\prime}$ as in the conclusion of Theorem 3 . We then consider the function $g^{\prime}$, a knot function $g^{\prime \prime}$ so that image

$$
g^{\prime \prime}=\left(J_{A}-A_{1} A_{2}\right) \cup\left(A_{1} B_{1} \cup B_{1} B_{2} \cup A_{2} B_{2}\right),
$$

and the disk $B_{1} B_{2} A_{2}$. Again, we obtain a sequence as in Theorem 3 . The next disk considered is $A_{2} B_{2} A_{3}$, etc. Thus we obtain a sequence of knot functions $k_{0}=g_{A}$, $k_{1}, \ldots, k_{r}=g_{B}$ so that $k_{i+1}$ is obtained from $k_{i}$ by a simple transformation. If we do this for each $a_{p}, 0 \leqq p<k$, then it is evident that we can produce a sequence $g_{0}^{\prime}, \ldots, g_{n}^{\prime}$ as in the conclusion of the theorem. Remembering now that the point $A^{\prime}$ involved previously was not necessarily the origin, we see from the following how the sequence $g_{0}^{\prime}, \ldots, g_{n}^{\prime}$ defined above can be used to define a new sequence $g_{0}, \ldots, g_{n}$, where the boundary collections are the usual ones relative to the $x y$ plane.

We use a small rotation $V$ of $E^{3}$ so that the center of $S$ is fixed, $A^{\prime}$ is moved to the origin, and no 0 -simplex of $T_{i}(i=0,1)$ is moved as much as $\varepsilon / 2$. We let $g_{p}=V g_{p}^{\prime}$, $p=0, \ldots, n$, and let $v(K)=K^{\prime}$ and $v(L)=L^{\prime}$. Now using new approximations of 
0 -simplexes new knots $K^{\prime \prime}$ and $L^{\prime \prime}$ may be determined so that (1) $K^{\prime \prime}$ is near $K, K^{\prime}$ and $L^{\prime \prime}$ is near $L, L^{\prime}$ and (2) some new sequences of knot functions may be defined as in the case of $J_{A}$ and $J_{B}$ above for the pairs $\left(K, K^{\prime \prime}\right),\left(K^{\prime \prime}, K^{\prime}\right),\left(L^{\prime}, L^{\prime \prime}\right)$ and $\left(L^{\prime \prime}, L\right)$. The knot functions obtained in these four cases are used together with" $g_{0}, \ldots, g_{n}$ to give the desired sequence.

8. Matrix representations. In this section we give a way of associating with a knot function $f:[0,1) \rightarrow K$ an $n+2$ by $2 n$ matrix $M(K)$, where $\pi K$ has $n$ double points and each entry of $M(K)$ is a 0,1 or -1 .

We start labeling the columns across the top using the word $W(f)$ of $f$. The rows represent in a one-to-one way the elements of the boundary collection, $C(f)$ or equivalently, the complementary domains. For example, if row $i$ corresponds to $C_{1} \in C(f)$ and $B=\left(\pi f\left(a_{p}\right)\right)^{e}\left(\pi f\left(a_{p+1}\right)\right)^{e_{p+1}} \in C_{1}$ then $M_{i q}=e_{q}$ for $q=p, p+1$. Any other places in row $i$ not filled in this way are filled with zeros. A matrix for the trefoil knot $T$ of Figure 1 is

$$
M=\left\{\begin{array}{rrrrrr}
a & b^{-1} & c & a^{-1} & b & c^{-1} \\
1 & -1 & 1 & -1 & 1 & -1 \\
1 & -1 & 0 & -1 & 1 & 0 \\
1 & 0 & 1 & -1 & 0 & -1 \\
0 & -1 & 1 & 0 & 1 & -1 \\
1 & -1 & 1 & -1 & 1 & -1
\end{array}\right\}
$$

Also, it seems to be convenient to think of two of the matrices as being equivalent if one is obtained from the other by

(0) the identity transformation,

(1) any cyclic permutation of columns,

(2) reversal of all signs,

(3) any permutation of rows,

(4) reversal of order of columns, or

(5) any operation corresponding to a simple transformation.

With the aid of Theorems 1 and 4, it is not hard to show

THEOREM 14. If $M(K)$ is equivalent to $M(L)$ then $K$ is isomorphic to $L$.

\section{REFERENCES}

1. R. H. Bing, An alternative proof that 3-manifolds can be triangulated, Ann. of Math. (2) 69 (1959), 37-65. MR 20 \#7269.

2. R. H. Crowell and R. H. Fox, Introduction to knot theory, Ginn, Boston, Mass., 1963. MR 26 \#4348.

3. C. F. Gauss, Werke (8), Teubner, Leipzig, 1900, pp. 272, 282-286.

4. W. Graeub, Die semilinearen Abbildungen, S.-B. Heidelberger Akad. Wiss. Math.-Nat. K1. 1950, 205-272. MR 13, 152.

5. M. L. Marx, The Gauss realizability problem, Proc. Amer. Math. Soc. 22 (1969), 610-613. MR 39 \#6297. 
6. E. E. Moise, Affine structures in 3-manifolds. VIII. Invariance of the knot types; local tame embedding, Ann. of Math. (2) 59 (1954), 159-170. MR 15, 889.

7. R. L. Moore, Foundations of point set theory, rev. ed., Amer. Math. Soc. Colloq. Publ., vol. 13, Amer. Math. Soc., Providence, R. I., 1962. MR 27 \#709.

8. J. V. Sz.-Nagy, Uber ein topologisches Problem von Gauss, Math. Z. 26 (1927), 579-592.

9. L. P. Neuwirth, Knot groups, Ann. of Math. Studies, no. 56, Princeton Univ. Press, Princeton, N. J., 1965. MR 31 \#734.

10. D. E. Penney, An algorithm for establishing isomorphism between tame prime knots in $E^{3}$, Dissertation, Tulane University, New Orleans, La., 1965.

11. - A knot projection has four small complementary domains (unpublished).

12. D. E. Sanderson, Isotopy of 3-manifolds. I. Isotopic deformations of 2-cells and 3-cells, Proc. Amer. Math. Soc. 8 (1957), 912-922. MR 19, 760.

13. - Isotopy in 3-manifolds. II. Fitting homeomorphisms by isotopy, Duke Math. J. 26 (1959), 387-396. MR 21 \#5956.

14. - Isotopy in 3-manifolds. III. Connectivity of spaces of homeomorphisms, Proc. Amer. Math. Soc. 11 (1960), 171-176. MR 22 \#2986.

15. P. G. Tait, On knots, Scientific Paper, I, Cambridge Univ. Press, London, 1898.

16. L. B. Treybig, A characterization of the double point structure of the projection of a polygonal knot in regular position, Trans. Amer. Math. Soc. 130 (1968), 223-247. MR 36 \#878.

17. —-, Prime mappings, Trans. Amer. Math. Soc. 130 (1968), 248-253. MR 36 \#879.

\section{TUlANE UNIVERSITY, New OrLeans, Louisiana 70118}

